\title{
Linx
}

Revue des linguistes de l'université Paris X Nanterre

45 | 2001

Invariants et variables dans les langues. Études typologiques

\section{Les systèmes de classes nominales des langues Niger-Congo : prototype et variations}

\section{Denis Creissels}

\section{(2) OpenEdition}

\section{Journals}

Édition électronique

URL : http://journals.openedition.org/linx/837

DOI : $10.4000 /$ linx. 837

ISSN : 2118-9692

Éditeur

Presses universitaires de Paris Nanterre

Édition imprimée

Date de publication : 1 décembre 2001

Pagination : 157-166

ISSN : 0246-8743

Référence électronique

Denis Creissels, « Les systèmes de classes nominales des langues Niger-Congo : prototype et variations », Linx [En ligne], 45 | 2001, mis en ligne le 25 juin 2012, consulté le 10 décembre 2020. URL : http://journals.openedition.org/linx/837 ; DOI : https://doi.org/10.4000/linx.837

Ce document a été généré automatiquement le 10 décembre 2020.

Département de Sciences du langage, Université Paris Ouest 


\title{
Les systèmes de classes nominales des langues Niger-Congo : prototype et variations ${ }^{1}$
}

\author{
Denis Creissels
}

1 On trouve dans presque toutes les familles de langues constituant le phylum Niger-Congo (qui regroupe la majorité des langues parlées en Afrique Noire) une proportion plus ou moins importante de langues attestant le type particulier de classification nominale ${ }^{2}$ connu sous le nom de "classes nominales ", ou du moins des vestiges d'un système de ce type. Les langues les plus typiques de ce point de vue sont les langues bantoues et les langues atlantiques.

2 Comme les autres types de classification nominale couramment désignés comme " systèmes de classes nominales ", les systèmes de classes nominales des langues NigerCongo sont typologiquement à la fois proches des systèmes de "genre » et nettement différents des systèmes de "classificateurs » : comme le "genre », ils mettent en jeu de manière cruciale des mécanismes d'accord fortement grammaticalisés qui n'ont pas leur équivalent dans les systèmes de "classificateurs ${ }^{3}{ }^{3}$ Mais il semble qu'on ne trouve nulle part ailleurs des systèmes de classification nominale identiques jusque dans les détails à ceux des langues Niger-Congo. Il peut donc être intéressant (et c'est l'objectif de cet article) de proposer une caractérisation qui permette une comparaison plus fine avec l'ensemble des systèmes décrits comme systèmes de «classes nominales » ou de " genres ». Les exemples illustratifs seront tirés du tswana ${ }^{4}$, dont le système de classes est très proche du prototype qu'on peut dégager de la comparaison des langues Niger-Congo, permettant ainsi à la fois d'illustrer le prototype et de faire apparaître les écarts, variations et irrégularités qui se manifestent même dans des les langues dont le système de classes s'écarte relativement peu du prototype Niger-Congo. 


\section{Les systèmes de classes nominales Niger-Congo comme systèmes d'accord fortement grammaticalisés dans lesquels il est impossible de dissocier marques de genre et marques de nombre}

3 Les traits essentiels des systèmes de classes nominales Niger-Congo sont les suivants :

4 (a) les noms doivent être répartis en un nombre déterminé de sous-ensembles pour rendre compte de mécanismes d'accord entre le nom et ses modifieurs, entre le nom et les pronoms qui le représentent, et enfin entre le nom et le verbe dont il est un argument ;

(b) les mots impliquées dans ces mécanismes d'accord (noms, modifieurs de nom, pronoms et verbes) portent obligatoirement des affixes (les marques de classe) qui déterminent leur comportement du point de vue de l'accord ;

(c) tous les noms sont concernés par la classification, qui est fondamentalement une classification des noms plutôt qu'une classification qui prendrait directement en compte les référents des noms : l'affectation d'un nom renvoyant à un type donné de référent est lexicalisée au sens où les locuteurs n'ont pas la liberté de faire varier la marque de classe du nom, comme cela peut se produire dans les systèmes de «classificateurs », pour faire ressortir divers traits sémantiques d'un même référent. ${ }^{5}$

5 Sémantiquement, la fonction la plus évidente des marques de classe (qui n'est toutefois pas la seule, comme nous le verrons) est d'encoder la distinction singulier / pluriel : dans ces langues, la distinction de nombre s'exprime tout aussi systématiquement que dans les langues d'Europe, mais il n'est jamais possible d'isoler des affixes exprimant spécifiquement le nombre.

6 La correspondance entre les distinctions de classe au singulier et les distinctions de classe au pluriel donne souvent lieu à des irrégularités et à des variations. Ceci explique que beaucoup de descriptions de ces systèmes ne mettent pas en avant la possibilité de répartir les lexèmes nominaux en genres (dont l'inventaire précis est souvent problématique), mais prennent plutôt comme point de départ une répartition des formes nominales en classes dans laquelle la forme de singulier et la forme de pluriel d'un même nom sont traitées comme deux unités distinctes, la notion de genre étant ultérieurement introduite comme couple de classes qui se correspondent dans l'expression du nombre. Par exemple, le nom tswana pour «femme » appartient au genre $1 / 2$, ce qui signifie que sa forme de singulier gouverne les accords de la classe 1 , tandis que sa forme de pluriel gouverne les accords de la classe $2 .^{6}$

7 Les exemples (1) à (4) illustrent sur l'exemple du tswana cette caractérisation des systèmes de classification nominale Niger-Congo.

(1) La répartition des formes nominales du tswana en 12 classes d'accord illustrée par l'accord des adjectifs

\begin{tabular}{|l|l|l|l|}
\hline a. & cl. 1 & mo-sadi yo mo-ša & 'nouvelle femme' \\
\hline b. & cl. 2 & ba-sadi ba ba-ša & 'nouvelles femmes' \\
\hline
\end{tabular}




\begin{tabular}{|l|l|l|l|}
\hline c. & cl. 3 & mo-lemo o mo-ša & 'nouveau médicament' \\
\hline d. & cl. 4 & me-lemo e me-ša & 'nouveaux médicaments' \\
\hline e. & cl. 5 & le-saka le le-ša & 'nouvel enclos' \\
\hline f. & cl. 6 & ma-raka a ma-ša & 'nouveaux enclos' \\
\hline g. & cl. 7 & se-kolo se se-ša & 'nouvelle école' \\
\hline h. & cl. 8 -10 & di-kolo tse din-tšha & 'nouvelles écoles' \\
\hline & & di-kgosi tse din-tšha & 'nouveaux chefs' \\
\hline i. & cl. 9 & Ø-kgosi e n-tšha & 'nouveau chef' \\
\hline j. & cl. 11 & lo-kwalo lo lo-ša & 'nouveau livre' \\
\hline k. & cl. 14 & bo-jang jo bo-ša & 'nouvelle herbe' \\
\hline l. & cl. 15-17 & go lema mo go-ša & 'nouvelle façon de cultiver' \\
\hline
\end{tabular}

(2) L'accord de noms de cl. 1, 5, 7 et 9 (ou de leur pluriel, lorsque le sens du modifieur l'exige) avec divers types de modifieurs

\begin{tabular}{|c|c|c|}
\hline a. & mo-sadi w-a motse & 'femme du village' \\
\hline & le-kau l-a motse & 'garçon du village' \\
\hline & se-fofu s-a motse & 'aveugle du village' \\
\hline & $\emptyset$-ngaka y-a motse & 'médecin du village' \\
\hline b. & mo-sadi yo-le & 'cette femme-là' \\
\hline & le-kau le-le & 'ce garçon-là' \\
\hline & se-fofu se-le & 'cet aveugle-là' \\
\hline & $\varnothing$-ngaka e-le & 'ce médecin-là' \\
\hline c. & mo-sadi o-fe? & 'quelle femme?' \\
\hline & le-kau le-fe? & 'quel garçon?' \\
\hline & se-fofu se-fe? & 'quel aveugle?' \\
\hline
\end{tabular}




\begin{tabular}{|c|c|c|}
\hline & $\varnothing$-ngaka e-fe? & 'quel médecin?' \\
\hline d & mo-sadi o-sele & 'une autre femme' \\
\hline & le-kau le-sele & 'un autre garçon' \\
\hline & se-fofu se-sele & 'un autre aveugle' \\
\hline & Ø-ngaka e-sele & 'un autre médecin' \\
\hline e. & mo-sadi o-pe & 'aucune femme' \\
\hline & le-kau le-pe & 'aucun garçon' \\
\hline & se-fofu se-pe & 'aucun aveugle' \\
\hline & $\emptyset$-ngaka e-pe & 'aucun médecin' \\
\hline f. & ba-sadi b-otlhe & 'toutes les femmes' \\
\hline & ma-kau Ø-otlhe & 'tous les garçons' \\
\hline & di-fofu ts-otlhe & 'tous les aveugles' \\
\hline & di-ngaka ts-otlhe & 'tous les médecins' \\
\hline g. & mo-sadi mo-ngwe & 'une femme' \\
\hline & le-kau le-ngwe & 'un garçon' \\
\hline & se-fofu se-ngwe & 'un aveugle' \\
\hline & Ø-ngaka n-ngwe & 'un médecin' \\
\hline h. & ba-sadi ba le pedi & 'deux femmes' \\
\hline & ma-kau a le pedi & 'deux garçons' \\
\hline & di-fofu di le pedi & 'deux aveugles' \\
\hline & di-ngaka di le pedi & 'deux médecins' \\
\hline i. & \multicolumn{2}{|c|}{ mo-sadi yo ke mo thusitseng maabane } \\
\hline
\end{tabular}




\begin{tabular}{|l|l|}
\hline & 1-femme jonctcl1 s1s ocl1 avoir+aidé.rel hier \\
\hline & 'la femme que j'ai aidée hier' \\
\hline & le-kau le ke le thusitseng maabane \\
\hline & le garçon que j'ai aidé hier' \\
\hline se-fofu se ke se thusitseng maabane \\
\hline & 7- aveugle jonctcl7 s1s ocl7 avoir+aidé.rel hier \\
\hline & 'l'aveugle que j'ai aidé hier' \\
\hline \hline & $\varnothing$-ngaka e ke e thusitseng \\
\hline & 9- médecin jonctcl9 s1s ocl9 avoir+aidé.rel hier \\
\hline & 'le médecin que j'ai aidé hier' \\
\hline
\end{tabular}

(3) La répétition obligatoire des marques de classe sur tous les modifieurs du nom dans un groupe nominal complexe

\begin{tabular}{|l|}
\hline a. mo-sadi yo mo-leele yo mo-ntsho \\
\hline 1-femme jonctcl1 1-grand jonctcl1 1-noir \\
\hline yo o opelang yo-le \\
\hline jonctcl1 scl1 chanter.rel 1-dém \\
\hline 'cette femme de haute taille et au teint foncé qui chante' \\
\hline b. le-kau le le-leele le le-ntsho \\
\hline 5-garçon jonctcl5 5-grand jonctcl5 5-noir \\
\hline le le opelang le-le \\
\hline jonctcl5 scl5 chanter.rel 5-dém \\
\hline 'ce garçon de haute taille et au teint foncé qui chante' \\
\hline
\end{tabular}

(4) L'accord en classe entre le nom et les pronoms ou indices pronominaux dont il peut être l'antécédent 


\begin{tabular}{|l|}
\hline a. Mo-sadi o lapile 'La femme est fatiguée' \\
\hline \hline 1-femme scl1 être+fatigué \\
\hline Le-kau le lapile 'Le garçon est fatigué' \\
\hline Se-fofu se lapile 'L'aveugle est fatigué' \\
\hline Ø-Ngaka e lapile 'Le médecin est fatigué' \\
\hline b. Ke mo thusitse 'Je l'ai aidée (la femme)' \\
\hline s1s ocl1 avoir+aidé \\
\hline \hline Ke bua le yone 'Je parle avec lui (le médecin)' \\
\hline Ke le thusitse 'Je l'ai aidé (le garçon)' \\
\hline Ke se thusitse 'Je l'ai aidé (l'aveugle)' \\
\hline Ke e thusitse 'Je l'ai aidé (le médecin)' \\
\hline c. Ke bua le ene 'Je parle avec elle (la femme)' \\
\hline \hline s1s parler avec procl1 \\
\hline Ke bua le lone 'Je parle avec lui (le garçon)' \\
\hline \hline
\end{tabular}

\section{Le prototype et les écarts par rapport au prototype}

Dans la section précédente ont été résumées les caractéristiques généralement considérées comme typiques des systèmes de classes nominales Niger-Congo. Mais aucune langue Niger-Congo n'a un système qui pourrait être considéré sans aucune réserve comme la réalisation la plus simple possible du prototype idéal rendant compte des principales régularités qui se dégagent de la comparaison.

9 En ce qui concerne les aspects formels de la classification nominale, toutes les langues à classes de la famille Niger-Congo s'écartent en effet plus ou moins de la situation idéalement simple dans laquelle toute forme impliquée dans un mécanisme d'accord 
présenterait invariablement un marqueur de classe (a) explicite, (b) identique pour tous les noms ayant ayant les mêmes propriétés d'accord, (c) non ambigu, (d) dépourvu de variations allomorphiques et (e) toujours obligatoire. Les exemples tswana ci-dessus permettaient déjà d'entrevoir que, même dans un système qui s'écarte relativement peu du prototype idéal :

10 (a) L'appartenance du nom à une classe n'est pas toujours explicitement indiquée par un affixe. Certains noms peuvent être invariablement dépourvus d'affixes de classe. Par exemple, en bantou, un préfixe ${ }^{*} n$ - de classe 9 peut être reconstruit, mais dans les langues bantoues actuelles, les formes nominales de classe 9 sont souvent dépourvues de préfixe. Qui plus est, les cas de noms qui ont en principe un affixe de classe mais dont l'affixe de classe peut dans certaines conditions ne pas apparaitre, sans être fréquents, ne sont pas totalement inconnus ; par exemple, en tswana - ex. (5), les préfixes des noms de classe 5, 7, 8-10, 11 et 14 peuvent être omis si l'accord avec un autre mot rend apparente la classe à laquelle appartient le nom.

\begin{tabular}{|l|}
\hline (5) a. le-saka 'enclos' \\
\hline 5-enclos \\
\hline \\
\hline le-saka l-a dikgomo = saka l-a dikgomo \\
\hline 5-enclos 5-gén 10vache enclos 5-gén 10cattle \\
\hline 'enclos à vache' \\
\hline b. di-kgomo 'vaches' \\
\hline 10-vache \\
\hline \\
\hline di-kgomo ts-a kgosi = kgomo ts-a kgosi \\
\hline 10-vache 10-gén 9chef vache 10-gén 9chef \\
\hline 'vaches du chef' \\
\hline
\end{tabular}

11 (b) Deux noms qui ont les mêmes propriétés d'accord peuvent porter des marques de classe différentes. Certains descripteurs considèrent dans de tels cas que deux classes peuvent se distinguer dans la morphologie nominale et se confondre totalement au niveau des accords. Mais une telle décision est logiquement criticable, car elle remet en question la cohérence même de la notion de classe, et dans la pratique, elle a l'inconvénient de permettre une prolifération des « classes » difficile à contrôler, car elle offre une solution de facilité dans les cas (fréquents) où un même marqueur de classe a des allomorphes qui ne se ramènent pas facilement à une forme phonologique abstraite unique. L'autre solution est de considérer que des noms qui ont les mêmes accords mais 
des affixes de classe différents appartiennent à deux sous-classes d'une même classe. C'est ainsi qu'on reconnaît en bantou une sous-classe 1a de la classe 1 constituée par des noms qui ont les accords de la classe 1 , mais qui ont un préfixe vide au lieu du préfixe $m u$ qu'ont la plupart des noms de la classe 1 .

(c) Deux noms portant des marques de classe identiques peuvent avoir des propriétés d'accord différentes. Dans de tels cas, on considère généralement qu'on a des classes distinctes avec une homonymie au niveau des affixes du nom. Cette situation, qui est celle des classes bantoues 1 et 3 (cf. ex. (1) ci-dessus) est importante pour l'évolution de ces systèmes, car l'homonymie au niveau des affixes du nom peut favoriser une confusion dans leurs propriétés d'accord, et aboutir finalement à la fusion de deux classes à l'origine distinctes ; ceci s'observe en particulier lorsque deux classes ont en commun l'absence de préfixe nominal explicite. ${ }^{7}$

13 (d) Les marques de classe participant à un même schème d'accord (c'est-à-dire les affixes qui indiquent la même classe sur des mots de nature différente - noms, modifieurs de noms, pronoms ou verbes) sont loin d'être toujours phonologiquement uniformes. Par exemple, en tswana, les exemples ci-dessus suffisent à constater que les classes 1, 3, 4, 6, 9 et 15-17 mettent en jeu des marques phonologiquement hétérogènes. Une telle situation peut résulter de la confusion de classes originellement distinctes (chacune ayant fourni une partie du matériau morphologique de la classe résultant de la fusion), mais synchroniquement, une fois qu'une telle situation s'est stabilisée, les règles d'accord s'appliquent avec la même régularité et la même rigueur quel que soit le degré d'hétérogénéité phonologique de l'ensemble des marques mises en jeu.

14 (e) La présence de certaines marques de classe peut être liée à un conditionnement discursif. Ceci ne se produit jamais au niveau des modifieurs du nom (à partir du moment où un modifieur est apte à s'accorder en classe avec le nom, l'accord est toujours obligatoire), mais les choses sont différentes au niveau de l'accord du verbe avec ses arguments: dans beaucoup de langues, l'accord obligatoire du verbe avec son sujet contraste avec le caractère facultatif de l'indexation de l'objet, qui dépend de conditions discursives, comme dans l'ex. tswana (6).

\begin{tabular}{|l|}
\hline (6) a. Mo-sadi o lapile 'La femme est fatiguée' \\
\hline 1- woman scl1 être+fatigué \\
\hline \\
\hline O lapile ‘Elle est fatiguée' \\
\hline scl1 être+fatigué \\
\hline *Mo-sadi lapile \\
\hline \\
\hline b. Ke thusitse mo-sadi 'J'ai aidé la femme’ \\
\hline s1s avoir+aidé 1-femme \\
\hline
\end{tabular}


Ke mo thusitse 'Je l'ai aidée'

s1s ocl1 avoir+aidé

\section{Aspects sémantiques des systèmes de classes nominales Niger-Congo}

Les aspects sémantiques de la classification nominale Niger-Congo soulèvent des problèmes beaucoup plus délicats que les aspects strictement formels, tant au niveau descriptif que théorique. Des présentations élémentaires ont popularisé l'idée que les classes nominales Niger-Congo ont des valeurs sémantiques simples et évidentes, mais les choses sont plus complexes, et en définitive, la question de la motivation sémantique des classes Niger-Congo se pose à peu près dans les mêmes termes que la question de la motivation sémantique du genre indo-européen: l'assignation des noms à une classe n'est, ni totalement arbitraire du point de vue sémantique, ni conditionnée par des notions comme "animaux", "personnes", "arbres" ou "parties du corps» aussi simplement que le suggèrent beaucoup de descriptions. ${ }^{8}$

Dans la plupart des langues Niger-Congo à classes nominales, la seule distinction sémantique reliée de manière totalement évidente au système formel de classes d'accord est thumain. En particulier, les noms communs se référant à des humains peuvent être répartis dans plusieurs genres, mais les noms propres de personnes humaines ont tous les même accords indépendamment de leur morphologie. Et même dans des langues où au niveau phrastique les règles d'accord tiennent exclusivement compte des marques de classe portées par les noms, l'accord en classe des anaphoriques par contre ne fonctionne strictement que si l'anaphorique est à proximité immédiate de son antécédent: l'ex. tswana ( $7 \mathrm{~b})$ montre que les anaphoriques renvoyant à des référents humains, s'ils sont relativement éloignés de leur antécédent, peuvent être pris dans les classes 1 (sg) / 2 (pl.) indépendamment de la classe à laquelle appartient leur antécédent.

\begin{tabular}{|c|}
\hline (7) a. Ke bonye Ø-ngaka le b-ana b-a yone \\
\hline s1s avoir+vu 9-médecin avec 2-enfant 2-gén procl9 \\
\hline 'J'ai vu le médecini avec sesi enfants (litt. avec les enfants de luii)' \\
\hline b. Ke bonye $\varnothing$-ngaka e n-tšha \\
\hline s1s avoir+vu 9-médecin jonctcl9 9-nouveau \\
\hline ko $\quad$-toropo-ng, e bile ke buile le ene \\
\hline prép 9-ville-loc et s1s avoir+parlé avec procl1 \\
\hline 'J'ai vu le nouveau médecini en ville, et j'ai parlé avec luii' \\
\hline
\end{tabular}


17 Pour le reste, les noms ayant certaines affinités sémantiques tendent indéniablement à se regrouper, mais il est difficile d'arrêter avec précision la liste des traits sémantiques pertinents, car ils sont souvent relativement abstraits, et leur relation à la répartition des noms en classes est souvent de nature non pas absolue mais statistique. L'observation du traitement des termes d'emprunt est de ce point de vue instructive.

Les noms empruntés peuvent être affectés à une classe sur la base de leur sémantisme, et parfois même de manière inattendue. Par exemple, en tswana, mo-fine "vin » (du néerlandais wijn) a été rangé dans la classe 3, ce qui n'est ni une affectation phonologiquement conditionnée, ni une affectation par défaut, la seule explication possible étant que, même s'ils ne sont pas au total très nombreux, on trouvait déjà parmi les noms de classe 3 plusieurs noms de substances psychoactives comme mo-re " médicament ou produit magique ", mo-tsoko «tabac", mo-tokwane " marijuana ». Mais ceci n'est pas très courant : la plupart du temps, l'affectation des termes d'emprunt à une classe se fait sur une base morpho-phonologique: ou bien l'initiale du nom emprunté peut être réinterprétée comme un préfixe de classe (comme bo-rotho pl. ma-rotho " pain ", du néerlandais brood, ou se-kepe pl. di-kepe «bateau ", du néerlandais schip), ou bien les noms empruntés sont affectés par défaut à la classe 9 , caractérisée par un préfixe vide.

19 La difficulté à déterminer jusqu'à quel point le sémantisme des noms permet de prédire la classe à laquelle ils appartiennent et le fait que l'assignation d'une classe aux termes empruntés est le plus souvent sémantiquement arbitraire ne doivent toutefois pas faire oublier que certains aspects de ces systèmes mettent en jeu des relations très claires entre certaines marques de classe et certains traits sémantiques.

20 Il y a tout d'abord ce que l'on appelle la valeur dérivationnelle des marques de classe : nous avons déjà vu que la fonction la plus évidente des commutations entre marques de classe est l'expression du nombre, mais beaucoup de bases nominales ont la possibilité de se combiner avec plus de deux marques de classe, avec des significations typiquement dérivationnelles: arbre / fruit, individuel / collectif, concret / abstrait, diminutif, augmentatif, etc. - ex. (8) et (9).

\begin{tabular}{|l|}
\hline (8) a. cl. 1 mo-sadi 'femme' \\
\hline cl. 2 ba-sadi 'femmes' \\
\hline cl. 7 se-sadi 'comportement féminin' \\
\hline cl. 9 Ø-tshadi 'foule de femmes' \\
\hline cl. 11 lo-sadi 'foule de femmes' \\
\hline cl. 14 bo-sadi 'féminité' \\
\hline b. cl. $3 \quad$ mo-retlwa 'arbre de l'espèce moretlwa' \\
\hline cl. 2 me-retlwa 'arbres de l'espèce moretlwa' \\
\hline cl. 9 Ø-thetlwa 'fruit de l'arbre moretlwa' \\
\hline
\end{tabular}




\begin{tabular}{|l|}
\hline cl. 10 di-thetlwa 'fruits de l'arbre moretlwa' \\
\hline cl. 11 lo-retlwa 'bosquet d'arbres moretlwa' \\
\hline (9) a. mo-sadi 'femme' (cl. 1) / bo-sadi 'féminité' (cl. 14) \\
\hline mo-nna 'homme' (cl. 1) / bo-nna 'virilité' (cl. 14) \\
\hline mo-loi 'sorcier' (cl. 1) / bo-loi 'sorcellerie' (cl. 14) \\
\hline b. mo-retlwa 'arbre moretlwa' (cl. 3) / Ø-thetlwa 'fruit du moretlwa' (cl. 9) \\
\hline mo-rula 'arbre morula' (cl. 3) / Ø-thula 'fruit du morula' (cl. 9) \\
\hline m-milo 'arbre mmilo' (cl. 3) / Ø-pilo 'fruit du mmilo' (cl. 9)
\end{tabular}

21 Il y a aussi des régularités dans le choix de la classe à laquelle appartiennent les noms dérivés de verbes, ou les adjectifs utilisés comme noms. En tswana, le préfixe des adjectifs utilisés par référence à un nom (que celui-ci soit présent ou sous-entendu) tient compte exclusivement de la classe à laquelle appartient formellement le nom en question. Mais avec certaines marques de classe, les adjectifs peuvent avoir un fonctionnement nominal autonome; dans ce cas leur préfixe est choisi d'après le sens, et le choix est cohérent avec ce que l'on observe lorsque deux marques de classe peuvent commuter avec la même base nominale - ex. (10).

\begin{tabular}{|c|}
\hline (10) a. mo-sadi / ba-sadi (cl. 1 / cl. 2) 'femme(s)' \\
\hline bo-sadi (cl. 14) 'féminité' \\
\hline se-sadi (cl. 7) 'comportement féminin' \\
\hline b. -ntle 'bon, beau' (base adjectivale) \\
\hline bo-ntle (cl. 14) 'bonté, beauté' \\
\hline se-ntle (cl. 7) 'bien' \\
\hline
\end{tabular}

Plus généralement, les mots typiquement utilisés comme modifieurs de noms ou comme anaphoriques se référant à des noms ont aussi dans certaines classes des emplois «absolus » dans lequel il n'ont de lien d'aucune sorte (syntaxique ou anaphorique) avec un nom, et alors, au lieu que leur marque de classe soit déterminée par une règle formelle d'accord, elle véhicule directement une signification; en tswana, les marques de cl. 1/2 signifient alors directement "personne(s) », et les marques de classe $7 / 8$ signifient directement « chose $(\mathrm{s}) »-$ ex. (11). 


\begin{tabular}{|l|}
\hline (11) a. mo-ngwe 'quelqu'un' / se-ngwe 'quelque chose' \\
\hline 1-un 7-un \\
\hline b. o-pe 'personne' / se-pe 'rien' \\
\hline 1-aucun 7-aucun \\
\hline c. b-a motse 'les villageois'/ ts-a motse 'les affaires du village' \\
\hline 2-gén village 8-gén village \\
\hline
\end{tabular}

Dans la perspective d'une comparaison avec des systèmes moins grammaticalisés, il est intéressant de noter que cet emploi, dans lequel les préfixes de mots fonctionnant en principe comme modifieurs véhiculent directement des significations générales comme " personne », «chose ", « lieu » ou «manière ", peut être considéré comme cas-limite d'un phénomène rarement mentionné dans les grammaires descriptives, dans lequel la marque de classe d'un mot fonctionnant typiquement comme modifieur est interprétée comme se référant à un nom particulier, même si ce nom n'est, ni présent, ni susceptible d'être considéré comme discursivement saillant. Par exemple, en tswana, les marques de la classe 5 , dans un contexte où il n'y a aucune autre possibilité d'établir une relation anaphorique avec un autre nom de classe 5 , peuvent s'interpréter par défaut comme se référant à letsatsi «jour»: le le latelang «le suivant (cl.5)» peut renvoyer anaphoriquement à n'importe quel nom de classe 5 , mais si le contexte ne suggère aucune relation anaphorique, le le latelang est compris comme «le jour suivant», exactement comme si on disait explicitement letsatsi le le latelang.

\section{Conclusion}

En guise de conclusion, il est intéressant de situer les systèmes de classes nominales Niger-Congo du point de vue des processus de grammaticalisation, car ceci peut être un paramètre à prendre en considération dans une typologie de la classification nominale.

Toutes les observations pertinentes vont dans le sens d'une origine très ancienne de ces systèmes. Le système reconstruit pour le proto-bantou est très semblable à ce qu'on trouve dans beaucoup de langues bantoues actuelles. Quant au Niger-Congo, il n'y a pour l'instant aucune réelle reconstruction d'une proto-langue à ce niveau, mais tous les spécialistes s'accordent pour dire qu'on ne trouve aucune indication qui irait dans le sens de la reconstruction d'un système de classification moins grammaticalisé. ${ }^{9}$ Les systèmes de classes nominales « incomplets » par rapport au prototype bantou sont très communs dans diverses branches du Niger-Congo, mais tout indique qu'ils ne sont pas à interpréter comme des systèmes de classe émergents, mais plutôt comme le résultat de la désintégration de systèmes plus anciens proches du prototype bantou. En particulier, il est permis de considérer comme probable l'hypothèse d'une origine lexicale des marques 
de classe, mais la comparaison des langues Niger-Congo n'apporte aucune possibilité de rapprochement étymologique entre lexèmes et marques de classe.

Donc, les systèmes de classes nominales Niger-Congo ne semblent avoir conservé aucune trace des stades de leur évolution dans lesquels nous pouvons imaginer qu'ils présentaient un degré moindre de grammaticalisation, et c'est dans d'autres familles de langues qu'il faudra chercher des données permettant de reconstituer l'origine de tels systèmes de façon autre que purement spéculative. Par contre, les données Niger-Congo illustrent abondamment les processus que des systèmes de classification nominale à un stade avancé de grammaticalisation peuvent subir :

- érosion phonétique des marques de classe des noms et création de nouvelles marques de classe par l'agglutination d'anciens déterminants aux noms ;

- modifications dans la répartition des noms en classes;

- réduction du nombre de classes par fusion de classes originellement distinctes;

- réduction de l'inventaire des constructions dans lesquelles le nom est impliqué dans un phénomène d'accord: dans une langue comme le tswana, il est tout simplement impossible de combiner un nom avec un modifieur de quelque sorte que ce soit (adjectif, démonstratif, indéfini, génitif, relative, numéral, etc.) sans expliciter sur le modifieur la classe à laquelle appartient le nom, mais dans d'autres langues, certains types de modifieurs échappent à l'accord en classe ; c'est parfois le cas du génitif, et parfois aussi les adjectifs se subdivisent en adjectifs accordés en classe et adjectifs invariables.

Il est particulièrement intéressant d'observer que des systèmes d'accord mettant en jeu un nombre élevé de genres dont la base sémantique n'est pas évidente peuvent se réorganiser pour aboutir à un système à deux genres directement basé sur la distinction thumain ou tanimé. Nous avons vu que, même dans les systèmes fonctionnant en principe très strictement sur une base formelle, ces traits tendent à intervenir directement dans l'accord des anaphoriques avec leurs antécédents. Cette tendance peut aboutir à des systèmes comme celui du swahili, dans lesquels l'accord des noms de personnes humaines (et éventuellement aussi d'animaux) ne tient plus compte de la marque de classe portée par le nom. Et l'évolution peut se poursuivre jusqu'à une réorganisation radicale du système, comme en igo ${ }^{10}$, où les anciens préfixes de classe des noms fonctionnent toujours comme marques de nombre, mais où il n'y a plus que quatre classes d'accord directement déterminées par les traits sémantiques animé / inanimé et singulier / pluriel. Autrement dit, les systèmes d'accord complexes et fortement grammaticalisés n'évoluent pas nécessairement en perdant de plus en plus leur motivation sémantique : ils peuvent aussi se remotiver en se simplifiant.

\section{BIBLIOGRAPHIE}

CONTINI-MORAVA, E. (1997). Noun classification in Swahili : a cognitive-semantic analysis using a computer database. In R.K. Herbert (ed), African linguistics at the crossroads : Papers from Kwaluseni. Köln : Rüdiger Köppe. 599-628. 
CORBETT, G. (1991). Gender. Cambridge : Cambridge University Press.

CRAIG, C. G. (ed.). (1986). Noun classification and categorization.Amsterdam : John Benjamins.

CREISSELS, D. (2000). « Genres » indo-européens et « classe nominales » Niger-Congo. Faits de

Langues 14. 177-184.

DIXON, R. M. W. (1986). Noun classes and noun classification in typological perspective. In C. G. Craig (ed.), Noun classification and categorization. Amsterdam : John Benjamins. 105-112.

GBLEM, H. M. (1995). Description systématique de l'igo. Thèse de doctorat de l'université Stendhal

(Grenoble).

GRINEVALD, C. 1999. Typologie des systèmes de classification nominale. Faits de Langues 14. 101-122.

Williamson, K. \& R. Blench (2000). Niger-Congo. In B. Heine \& D. Nurse (eds.), African languages, an introduction. Cambridge : Cambridge University Press. 11-42.

\section{NOTES}

1. Ce texte doit beaucoup aux échanges avec Colette Grinevald, que je tiens à remercier ici pour ses suggestions.

2. Pour une approche générale de la classification nominale, cf. notamment Craig 1986, Dixon 1986 et Grinevald 1999.

3. cf. Corbett 1991, Creissels 2000

4. Les abréviations suivantes apparaissent dans les gloses des exemples tswana: DEM= démonstratif, GEN = génitif, JONCTCLX = joncteur de classe X (il s'agit d'un mot accordé en classe, qui est historiquement un ancien démonstratif, mais qui synchroniquement a perdu sa valeur déictique et est devenu obligatoire pour introduire certains modifieurs du nom), $L O C=$ locatif, OCLX $=$ indice d'objet de classe $\mathrm{X}, \mathrm{PREP}=$ préposition, $\mathrm{PROCLX}=$ pronom de classe $\mathrm{X}, \mathrm{SCLX}=$ indice de sujet de classe $\mathrm{X}, \mathrm{S} 1 \mathrm{~S}=$ indice de sujet de $1^{\text {ère }}$ personne du singulier, $\mathrm{REL}=$ marque de la forme relative des verbes.

5. "Lexicalisé » ne doit pas être compris comme synonyme de "sémantiquement arbitraire ». La question de la motivation sémantique de la répartition des noms en classes sera envisagée plus loin. Par ailleurs, ce qui vient d'être dit ne signifie pas qu'il n'y a pas de variation possible sur les marques de classe des noms : des variations sont possibles, mais comme nous le verrons plus loin, outre la distinction de nombre, elles servent à exprimer des valeurs dérivationnelles, ce qui est un type différent d'opération sémantique.

6. La numérotation des classes des langues bantoues se réfère aux classes proto-bantoues reconstruites, qui sont au nombre d'une vingtaine ; par exemple, l'absence de "classe 12 » en tswana signifie qu'aucune des classes nominales du tswana n'est le réflexe de la classe $12 \mathrm{du}$ proto-bantou, et une numérotation comme «classe 8-10 " indique une classe qui résulte de la fusion de deux classes distinctes du proto-bantou

7. Par exemple en tswana, on observe des flottements dans l'affectation des noms d'animaux à la classe 9 ou à la classe 1a, qui ont toutes deux un préfixe nominal vide ; l'assignation à la classe 9 semble étymologiquement correcte, mais la confusion avec la classe 1a est favorisée par le fait que la classe 1 regroupe typiquement les noms de personnes humaines, et que les protagonistes des contes traditionnels sont des animaux humanisés dont les noms s'accordent en classe 1 plutôt que dans la classe à laquelle ils appartiennent en tant que noms d'animaux ordinaires.

8. Pour une analyse précise de la question dans le cadre d'une langue particulière, cf. notamment Contini-Morava 1997.

9. cf. notamment Williamson \& Blench 2000. 
10. cf. Gblem 1995.

\section{AUTEUR}

\section{DENIS CREISSELS}

Denis.Creissels@univ-lyon2.fr 